\title{
2 Zur Lektüre und Analyse von Kehlmanns wissenschaftshistorischem Roman
}

Zwei längere Anmerkungen $\mathrm{zu}$ den nachstehenden Analysen sind an dieser Stelle erforderlich. 1. Im Anschluss an die im systematischen Teil ausführlich beschriebene Rolle des Lesers gilt es daran zu erinnern, dass die Differenzen zwischen fiktionalem und historischem bzw. fiktionalem und wissenschaftshistorischem Diskurs im Roman zwar von Beginn an durch kalkuliert gesetzte indexikalische Zeichen markiert sind, ihre eigentliche Realisierung jedoch erst dadurch finden, dass sie vom Leser aktiviert, also entsprechend seines Unterscheidungsvermögens, entsprechend vor allem seiner (wissenschafts-)historischen Kenntnisse ausgestaltet und kontextualisiert werden. ${ }^{49}$ Diese Realisierungen, wie bruchstückhaft das im Leser mobilisierte Vorwissen auch immer sein mag, bleiben unabhängig vom weiteren Fortgang der Erzählung in der Lektüre präsent, d.h. ,Geschichte‘ in ihrer zeit-, wissenschafts- und individualgeschichtlichen Ausdifferenzierung wird gleichsam als eine Art im Lesergedächtnis gespeicherter Subtext stets ,mitgelesen‘. Selbst für den angenommenen Fall, Kehlmanns Roman würde lediglich einen der genannten historischen Diskurse entfalten, so wären mit dem komplexen, auch auf die anderen Diskurse verweisenden Index der Anfangssätze die im folgenden nicht-erzählten Diskurse dennoch als Leerstellen generiert, die vom Leser, sofern dieser über ein entsprechendes Subtext-Archiv verfügt, konkretisiert würden. Dem Leser wird damit von Beginn an der Status eines Miterzählers zugewiesen, der das NichtErzählte durch die ihm bekannten historischen Erzählungen ergänzt oder auch das erzählte Historische mit den ihm jeweils bekannten historischen Erzählungen (komplettierend, konkretisierend, kritisierend usw.) in Beziehung setzt.

Besteht nun die miterzählende Leistung des empirischen Lesers, wie angedeutet, zunächst darin, dass er den im Gedächtnis in welcher Form auch immer gespeicherten historischen Subtext von Anfang an aktiviert - und diese Aktivierung ist sowohl Effekt der indexikalischen Zeichen als auch Voraussetzung für das Funktionieren der indexikalisch etablierten Differenzen und letztlich für das Funktionieren der Gattung überhaupt -, so ist das Miterzählen in erster Linie als ein Mitlesen jener historischen Erzählungen zu charakterisieren, auf die der Index verweist und die durch ihn im Leser erinnert werden. Als Leser des Romans und als Leser des historischen Subtexts vollzieht der Leser sozusa-

49 Zum Folgenden vgl. Kapitel 2.2 „Lector doctus - die Rolle des Rezipienten“ in vorliegender Studie. 
gen eine Synchronlektüre, ${ }^{50}$ wobei er in der Lektüre des gespeicherten historischen Subtexts zugleich zu dessen Wiedererzähler wird, der das Wiedererzählte (und jedes Wiedererzählen ist auch ein anderes Erzählen) der Romanerzählung und damit sich selbst dem fiktionalen Erzähler des Romans unverzichtbar zur Seite stellt. Die Lektüre des Subtextes geschieht zum einen in Abhängigkeit davon, ob und wie der Roman im weiteren Verlauf auf diesen Subtext rekurriert (ob er es bei der anfänglichen historischen Deixis belässt, ob er weitere indexikalische Momente einstreut oder ob er die Deixis zum narrativen Plot ausgestaltet) - das ,Ob` und ,Wie“ der Referentialisierung steuert die Lektüre maßgeblich-, zum anderen in Abhängigkeit davon, ob und wie der Subtext im historischen Gedächtnis des Leser überhaupt, verfasst“ ist. Sieht man einmal vom extremen Fall einer völligen Abwesenheit des Subtextes ab (sie führt - und dies trifft auf den historischen ebenso wie auf den wissenschaftlichen Roman $\mathrm{zu}$ - zum Kollaps der Gattung), so wird er in jedem Leser in einer anderen Gestalt vorliegen; mag diese Pluralität auch durch eine gemeinsame Schnittmenge historischer Fakten einerseits, durch die begrenzte Tropik der Geschichtserzählungen andererseits eingeschränkt sein, so wird sie doch durch die je unterschiedlichen Aneignungs-, Deutungs- und Aktivierungsweisen ihre Pluralität beibehalten. Damit ist ein weiterer Aspekt angesprochen: Die Synchronlektüre von Roman und Subtext setzt voraus, dass der Subtext bereits als ein ,irgendwie‘ verfasster im historischen Gedächtnis des Lesers konstituiert ist, sie setzt also die bereits erfolgte Lektüre jener historischen Erzählungen voraus, deren Produkt der Subtext ist. ${ }^{51}$ Anders formuliert: Die anamnetische Lektüre - als solche ließe sich die synchron zur realen Lektüre des Romans verlaufende Lektüre des Subtextes beschreiben ${ }^{52}$ - bedarf $\mathrm{zu}$ ihrem Gelingen notwendig einer bereits real erfolgten Lektüre historischer Erzählungen oder Darstellungen. ${ }^{53}$

50 Vgl. hierzu auch Bernadette Malinowski u. Jörg Wesche: Synchrones Lesen. Mathematik und Dichtung bei Michael Wüstefeld und Daniel Kehlmann, in: Poetiken der Gegenwart. Deutschsprachige Romane nach 2000, hrsg. v. Silke Horstkotte u. Leonhard Herrmann, Berlin, Boston 2013, S. 139-154.

51 Zugrunde gelegt wird hier ein weiter Begriff von ,Lektüre‘, der vom faktischen Lesen über die Rezeption anderer Medien bis hin zum bloßen ,Hörensagen‘ reicht und buchstäblich alle Formen der Aneignung einschließt.

52 Der Subtext ist ein Erinnerungstext, der im historischen Gedächtnis des Lesers aufbewahrt und entsprechend abgerufen werden kann; er ist in der Regel sozusagen nur noch in potenzierter Form an das materielle Medium der Schrift gebunden.

53 Sofern diese nicht erfolgt ist, wird sie der Leser, provoziert durch die Referenzsignale des Romans, nachholen - nachholen müssen, will er den Roman im Sinne der Gattung poetica scientiae aktualisieren. Vgl. dazu die Ausführungen im systematischen Teil. 
Der empirische Leser - gleichsam individualisiertes pars pro toto des impliziten Lesers $^{54}$ - kommt letztlich also nicht umhin, sich jene intertextuelle Kompetenz $\mathrm{zu}$ erarbeiten, die notwendig ist, um das im Text virtuell in der Gestalt des impliziten Lesers angelegte Äquivalenzsystem - die Summe aller potentiellen Lektüren - wenigstens partiell zu realisieren.

2. Die Aneignung dieser intertextuellen Kompeten $z,{ }^{55}$ d.h. die gründliche Auseinandersetzung mit dem historischen Material, ${ }^{56}$ die $\mathrm{zu}$ einer angemessenen Erfassung der narrativen Transformationsstrategien und entsprechend $\mathrm{zu}$ einer angemessenen Beurteilung der daraus resultierenden ästhetikspezifischen Differenzen und Modifikationen ${ }^{57}$ unabdingbar ist, liegt den folgenden Studien nicht ,stillschweigend“ voraus, sondern wird ihrerseits dokumentiert. Zwei Gründe sind hierfür ausschlaggebend: Zum einen ist der Erwerb dieser Kompetenz, der sich konkret in ausführlichen wissenschaftshistoriographischen Exkursen niederschlagen wird, motiviert durch die vom Roman selbst evozierten hermeneutischen Fragen und damit als ein Effekt der im Roman angelegten

54 Der implizite Leser meint jenes transzendentale Strukturmodell, „das als im Text verankerte strukturierte Hohlform die Gesamtheit der Vororientierungen bezeichnet, ,die ein fiktionaler Text seinen möglichen Lesern als Rezeptionsbedingungen anbietet', und zugleich den Übertragungsvorgang beschreibt, durch den sich die Textstrukturen über die Vorstellungsakte in den Erfahrungshaushalt des Lesers übersetzen“ (Meinhard Winkens: Art. „Wirkungsästhetik“, in: Metzler Lexikon Literatur- und Kulturtheorie, S. 678-681, hier S. 680).

55 Deupmann betont ebenfalls, dass „die ironische Erzählerrede ihre Figuren als Gegenstände historischen Wissens“ voraussetze, da andernfalls die „Spannung zwischen historischer Person und fiktiver Figur“, also die „Differenz zwischen historisch beglaubigtem und erkennbar unzuverlässigem ,Wissen““ eingeebnet und „das Spiel mit der historiografischen Form funktionslos bliebe“ (Deupmann: Poetik der Indiskretion, S. 251).

56 Am Beispiel etwa der Geschichte der nicht-euklidischen Mathematik werde ich versuchen, das von Stiening erhobene, wenn auch aufwändig zu realisierende Postulat, nämlich den „wissenschaftsgeschichtlichen Kontext“ - und zwar zunächst „unabhängig von den Rezeptionsformen und -ergebnissen des literarischen Autors“ zu erschließen und darüber hinaus zu fragen, „welche Stellung jenes historische Wissen innerhalb des poetischen Gefüges einnimmt“ (Gideon Stiening: „Und das Ganze belebt, so wie das Einzelne, sei“. Zum Verhältnis von Wissen und Literatur am Beispiel von Goethes Die Metamorphose der Pflanzen, in: Literatur und Wissen. Theoretisch-methodische Zugänge, hrsg. v. Tilmann Köppe, Berlin, New York 2011 S. 192-213, hier S. 205).

57 Auch Vanderbeke weist darauf hin, dass die Erfassung von literarischen Transformationen eine „kreative Auseinandersetzung mit einem Einfluß voraussetzt“ (Dirk Vanderbeke: Theoretische Welten und literarische Transformationen. Die Naturwissenschaften im Spiegel der ,science studies' und der englischen Literatur des ausgehenden 20. Jahrhunderts, Tübingen 2004, S. 201). 
Appellstruktur aufzufassen. ${ }^{58}$ Diese Appellstruktur ist ihrerseits zwar das Ergebnis von literarischen Transformationen, die auf der Grundlage ausgiebiger mathematikgeschichtlicher und historischer Rekonstruktionen seitens des Autors vorgenommen wurden, sie ist darüber hinaus aber auch Effekt einer bereits in den wissenschaftlichen und wissenschaftshistoriographischen Quellen selbst angelegten Appellstruktur. Hierzu ein Beispiel: Im Kontext der historischen Entwicklung der nichteuklidischen Geometrie (vgl. Kapitel 3.4.1) erweist sich diese Appellstruktur konkret als Diskrepanz zwischen einer geglätteten wissenschaftlichen und wissenschaftshistoriographischen Oberflächenstruktur und einer brüchigen, widersprüchlichen und geradezu hermetisch verdunkelten Tiefenstruktur, als eine Diskrepanz, die sich überhaupt nur auf dem Weg eines vertieften Quellen-Studiums - zu dem vor allem auch die synoptischvergleichende Lektüre verschiedener historischer Dokumente und historiographischer Darstellungen gehört - ,entdecken' und entsprechend produktiv machen lässt. Erst ein solches Studium vermag die bis heute offenen Fragen nach der ,inneren' Geschichte der nichteuklidischen Geometrie, nach ihren tief in die Historie eingesenkten ,genetischen' Ursachen und Zusammenhängen, bloßzulegen, ferner über die vergleichende Lektüre der zumeist auf eindeutige Interpretation gerichteten wissenschaftsgeschichtlichen Studien das Ungeklärte und Ungeglättete als das Ungeklärte und Ungeglättete aufzudecken und solcherart die der Wissenschaft und ihrer Geschichte implizite Appell- und Interrogativstruktur offenzulegen. Der literaturanalytische Befund, so wird sich zeigen, nimmt sich dazu überraschenderweise konträr aus: Hier ist es umgekehrt die Fiktion, die dazu tendiert, eine von Unbestimmtheiten, Leerstellen, ,Hermetismen' und Widersprüchen geprägte Historie $\mathrm{zu}$ verallgemeinern, $\mathrm{zu}$ vereindeutigen und auf ihr mathematisches Substrat zu verkürzen. ${ }^{59}$

58 Es ist vielleicht auch Resultat dieser Appellstruktur, dass Ottmar Ette nach aller aus historischer Perspektive durchaus nachvollziehbaren Polemik gegen Kehlmanns Verfremdung des historischen Humboldt ausführt, dass ,zumindest ein Teil der Leserschaft Interesse [...] an den historischen Figuren entwickelt und sich im Falle Humboldts auf eine Entdeckungsreise durch die Werke eines der großen Autoren und Denken des 19. Jahrhunderts einläßt“ (Ottmar Ette: Alexander von Humboldt in Daniel Kehlmanns Welt, in: HiN - Humboldt im Netz. Internationale Zeitschrift für Humboldt-Studien [Potsdam - Berlin] XIII, 25 [2012], S. 34-40, hier S. 39. Online verfügbar unter http://www.uni-potsdam.de/u/romanistik/humboldt/hin/hin25/ ette_deu.htm).

59 Mit dieser ,Vereindeutigungstendenz‘ wird das, was Deupmann treffend als „epistemische Unzuverlässigkeit“ bezeichnet (Deupmann: Poetik der Indiskretion, S. 243) freilich nicht getilgt, sondern vielmehr gesteigert. 
Die gleichrangige Behandlung von literarischem Text und historischen ,Subtexten' zielt demzufolge nicht nur darauf, den literarischen Textphänomenen jeweils semantische oder funktionale Entsprechungen bzw. Abweichungen im historischen Material vergleichend gegenüberzustellen, ${ }^{60}$ sondern ebenso darauf, das in den Quellen selbst angelegte ästhetische und literarische Potential aufzudecken. ${ }^{61}$ Aus Sicht der Literaturwissenschaft werden derartige Rekonstruktionsversuche nicht selten als ,positivistisch“ etikettiert, doch steht zu fragen, ob derlei Einwände nicht maßgeblich in der Annahme gründen, dass das Archiv der Geschichte und Wissenschaftsgeschichte Texte konserviert, die von historisch weitgehend erwiesenen und entsprechend gesicherten Tatsachen berichten. Damit soll die Literaturwissenschaft zwar nicht dem Verdacht eines naiven Geschichts- und Naturwissenschaftsverständnisses ausgesetzt werden. Sehr wohl aber gilt es - und dies mit Blick auf die Literaturwissenschaft als einer geisteswissenschaftlichen Disziplin ebenso wie mit Blick auf die Naturwissenschaften - darauf hinzuweisen, dass eine unübersehbare Kluft zwischen der jeweiligen disziplinären Wissenschaftstheorie und der wissenschaftlichen Praxis besteht, die $\mathrm{zu}$ überwinden sich als weitaus schwieriger und langwieriger erweist als gemeinhin angenommen. So tendiert die naturwissenschaftliche Lehr- und Forschungspraxis trotz der inzwischen weithin akzeptierten wissenschaftstheoretischen Einsicht in die historisch-kulturelle Bedingtheit naturwissenschaftlicher Theorien, Methoden und Praktiken nach wie vor dazu, ihre

60 Dies schließt selbstredend auch solche „Transformation[en] oder Umcodierung[en]“ ein, die, Deupmann im Rekurs auf Werber sehr präzise beschreibt, durch die „Wissen zum ,Medium für Formen“" wird (Christoph Deupmann: Poetik der Indiskretion. Zum Verhältnis von Literatur und Wissen in Daniel Kehlmanns Die Vermessung der Welt, in: Die Unendlichkeit des Erzählens. Der Roman in der deutschsprachigen Gegenwartsliteratur seit 1989, hrsg. v. Carsten Rohde u. Hansgeorg Schmidt-Bergmann, Bielefeld 2013, S. 237-256, hier S. 241).

$61 \mathrm{Zu}$ Kehlmanns Umgang mit den Quellen vgl. ausführlich Doll, der den Roman detailliert als einen postmodernen historischen Roman untersucht. „Die Schilderung“, so Doll, „beschränkt sich nicht auf Deskription, sondern erfolgt auf Basis einer kritischen Auseinandersetzung mit den Quellen, deren Ergebnis in pointiert ironischer Perspektive präsentiert wird. Die historische Dimension des Romans ist demnach geprägt von einer Auseinandersetzung mit dem Material, dessen Wahrheitsgehalt angezweifelt wird“ (Doll: Umgang mit Geschichte, S. 245). Legitimiert werde dieses Misstrauen gegenüber dem Geschriebenen in der metahistorischen Dimension des Romans, die verdeutlicht, dass „Erinnerung und Wahrnehmung [...] nicht nur Limitierungen unterworfen, sondern geradezu unzuverlässig und ungeeignet [sind], um Ereignisse akkurat festzuhalten“ (ebd., vgl. näherhin S. 269-274; 287-299). Wie Doll, der sich in seinen vergleichenden Analysen auf Humboldt konzentriert, überzeugend nachweist, legt der Roman damit nicht nur den „Konstruktcharakter des Materials“ (S. 288) und dessen fragwürdigen Wahrheitsgehalt offen, sondern darüber hinaus auch die Bedingungen seines Zustandekommens. 
Erkenntnisse am Maßstab eines klassischen Wissenschaftsbegriffs (Objektivität, Allgemeingültigkeit, Notwendigkeit) zu beurteilen und entsprechend öffentlich zu kommunizieren. Umgekehrt ist in den Kultur- und Geisteswissenschaften die Neigung zu beobachten, diese die ,hard sciences' aufweichenden Ergebnisse der Wissenschaftsgeschichte und -theorie einerseits als den lang ersehnten ,Beweis‘ für die Affinitäten zwischen Geistes- und Naturwissenschaften dankbar zu übernehmen, andererseits jedoch eben diese Affinitäten gerade dann auszublenden und zu ignorieren, wenn es darum geht, die Vorzüge der eigenen Disziplin auf dem Rücken der nunmehr wiederum ,positivistisch' betrachteten ,fremden' Disziplinen aufzuzeigen oder aber wenn es, wie im konkret angesprochenen Fall, darum geht, das Spezifische der Literatur durch die Aktualisierung eines positivistischen Wissenschafts- und Wissenschaftsgeschichtsverständnisses hervorzuheben. ${ }^{62}$

62 Die Schwierigkeit, einen annähernd ,neutralen‘ Boden zu gewinnen und der Versuchung zu widerstehen, sich je nach situations- und interessebedingten Bedürfnissen aus dem einen oder anderen Argumentationsfundus zu bedienen, ist - und dies gilt es durchaus auch selbstkritisch anzumerken - nicht ohne weiteres zu meistern. Die dafür verantwortlichen nichtwissenschaftlichen wie wissenschaftlichen Gründe einer genaueren Analyse zu unterziehen, ist ein Desiderat, das letztlich nur von einer transdisziplinär ausgerichteten Wissenschaftstheorie zu erfüllen wäre. 